Paper

UDC $621.382 .3 .016: 629.113: 621.37 / .39$

\title{
A New Evaluation Method of Second-Breakdown Resistibility in Power Transistors for Automotive Use
}

\author{
By \\ Takao Sasayama \\ Member
}

\begin{abstract}
Summary
Silicon power transistors of the $n^{+}-p-n-n^{+}$type have been developed for automotive applications. In order to obtain highly reliable performance under the severe environmental conditions usual in such applications, the characteristics of power transistors having a high degree of second-breakdown resistibility were investigated and a method of evaluating such transistors was developed. A nondestructive method of evaluating resistibility to "thermalmode" second-breakdown that uses the characteristic change in the current gain $h_{F E}$ of a transistor has been developed and applied to optimize the design of devices for automotive applications. The resistibility to "thermal-mode" second-breakdown of power transistor chips in hybrid integrated circuits was evaluated with this new method. It became clear that the thermal conditions between a chip and its almina substrate could be optimized to improve the resistibility to "thermal-mode" second-breakdown. Many kinds of automotive equipment that includes power transistors with a high degree of second-breakdown resistibility has been put to practical use in actual automobiles.
\end{abstract}

\section{Introduction}

A variety of electronic equipment is now in practical use in automobiles. For example, electronic engine control systems with microprocessors are now bringing about great improvements in engine performance. These control systems are usually composed of several kinds of semiconductor devices, including a microprocessor, memories, functional LSIs and power tran. sistors. Most power transistors for such applications are now integrated in hybrid ICs, which are usually mounted in the engine compartment.

Such power transistors must be very reliable under high temperature operation and highly resistive to second-breakdown phenomena caused by transient high power dissipation or high voltage surges during switching of an inductive load. The environmental conditions common to automotive applications are as follows. Ambient temperature changes range from -40 to $120^{\circ} \mathrm{C}$. Several kinds of surge, with different energy and voltage values, are often applied to the devices. The peak voltage of these surges is up to Takoo Sasayamo is with Hitachi Research Lab., Hitachi, Ltd. Manuscript received Oct. 7, 1983, revised May 1,1984. several hundred volts. The transistors are also subjected to high humidity and high levels of vibration.

It is also desirable for power transistors to be easy to mount on a hybrid IC substrate, and to have characteristics of low saturation voltage during the "ON" stage. It is needless to say that smaller size and low cost are also very desirable requirements.

Among these requirements, resistibility to secondbreakdown phenomena under severe environmental conditions presents the most serious problems, because breakdown causes catastrophic device and system failure.

In automotive applications, power transistors are used to operate as an inductive load switch, as shown in Fig. 1. A surge voltage is applied to such a transistor when it turns the current through the load off because the stored energy of the inductive load is instantly released at that time. This released energy is usually absorbed by the transistor during the time that it takes to turn the transistor off in a voltage sustaining operation in which power is dissipated. However, when sufficient energy is released from the inductive load, second-breakdown phenomena can occur ${ }^{(1)(2)}$.

Second-breakdown phenomena are composed of

本稚は Section E (Trans. I. E. E. of Japan, Vol. 104, No. 11/12, Nov./Dec., p. 225) からの転载である。 
"current-" and "thermal-modes", as shown in Fig. (3). $^{(3)}$ If a transistor is operating with high power dissipation, it often begins highly conductive operations when power is supplied for longer than a critical time, which is called the "delay time". Such a breakdown is assumed to be a "thermal-mode" second-breakdown"(4).

Second-breakdown phenomena triggered during high voltage and low current operations are assumed to be caused by avalanche injection from the collector region to the $n$-region of a $n^{+}-p-n-n^{+}$bipolar transistor ${ }^{(5)}$. This breakdown mode is called "current-mode" second-breakdown.

As mentioned above, resistibility to second-breakdown phenomena must be guaranteed in power transistors for automotive applications. The determination of resistibility to second-breakdown has, however, been very difficult because some degradation occurs even during the measurement of resistibility and because resistibility is affected by the conditions of the heat flow between a transistor chip and its substrate.

These problems impressed us with the necessity of developing a nondestructive method of determining the degree of resistibility of transistors to secondbreakdown phenomena.

\section{Evaluation Method}

As mentioned above, both "thermal-mode" and "current-mode" second-breakdown can be occured if the amount of the resistibility to second-breakdown of transistors is not so high. However, transistors can be protected from "current-mode" second-breakdown caused by high voltage surges easily, employing some voltage limiter for a surge absorber. Therefore, an evaluation method for "thermal-mode" second-breakdown was investigated.

"Thermal - mode" second - breakdown phenomena occur when "hot-stops" are generated at the collectorbase junction under unstable temperature and current conditions $^{(6)}$. These hot-spots can cause degradation of second-breakdown resistibility or, in some cases, transistor failure $e^{(7) \sim(9)}$.

Therefore, we determined to devolop a technique to
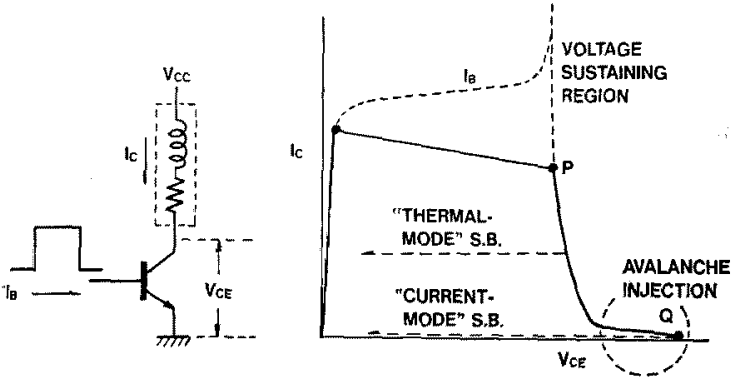

Fig. 1. Second-breakdown phenomena in automotive applications. detect hot-spots before they had completely formed. After investigating several methods of detection, we discovered the following phenomena.

In the characteristics of $V_{B E}$ versus $I_{B}$ which were observed by increasing the amplitude of the pulsed voltage applied between the collector and the emitter, while keeping the operating point of the transistor to a constant value, several remakable waveforms hav. ing a " $U$ " shape can be obsrved in the high power operation region ${ }^{(10)(11)}$.

After analyzing this phenomena, it was concluded that it is caused by changes in the current gain.

It is well known that the current gain of a transistor depends on ambient temperature and current density. Current gain increases with ambient temperature and decreases with current density for the greater part of the value of current density. When current density is low, current gain increases with current density, and current gain is unaffected by temperature at high current densities. At very high current densities, the dependence of current gain on temperature changes to negative, that is, it changes to the inverse characteristics of current gain at low current densities.

Assuming that current gain will change during the formation of hot-spots and as thermal instability increases, the circuit shown in Fig. 2 was developed to measure the second-breakdown resistibility of transistors through detection of variations in current gain.

It is desirable to keep the collector voltage and current constant when pulsed power is being dissipated at the transistor, because the power disspation, which effects junction temperature rise and current gain, can be kept constant.

In this circuit, collector-base voltage is held constant by a Zener diode connected between the collector and base electrodes. The collector current is also held constant as follows:

$$
I_{C}=\left(E-V_{Z D}\right) / R_{L}
$$

where,

$E$ : the output voltage of the pusled power supply, $V_{Z D}$ : the breakover voltage of Zener diode,

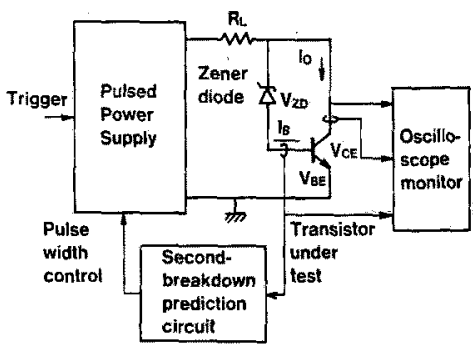

Fig. 2. Circuit configuration for measuring the second-breakdown resistibility of transistors through detection of variations in current gain. 
$R_{L}:$ the load resistance.

Consequently, the operating voltage and current conditions of the transistor under test are fixed regardless of the individual characteristics of the sample. Power dissipation is also held constant if the output voltage of the pulse supply is considered to be constant. The electrical or thermal phenomena which occur at the collector junction under these conditions will cause some variations in the base current or base-emitter voltage. Since the collector current is determined by external conditions, time variations in the current gain appear as the reciprocal of the base current.

As shown in later, the initiation of second-breakdown can be predicted by the change of the waveform of the base current. Second-breakdown prediction circuit in Fig. 2 detects the change of the waveform, and shuts off the pulsed power source immediately.

\section{Experimental}

Typical waveforms for the collector current, the collector emitter voltage, and base current observed in the circuit of Fig. 2, during high power operation are shown in Fig. 3. Where, the second-breakdown prediction circuit was removed. Therefore, the waveforms show the process to reach the initiation of the secondbreakdown.

These waveforms show that the operating point of a sample transistor does not change during high power operation, but that the base current changes characteristically.

The base current starts to decrease gradually from the initial values, reaches a minimum after a short

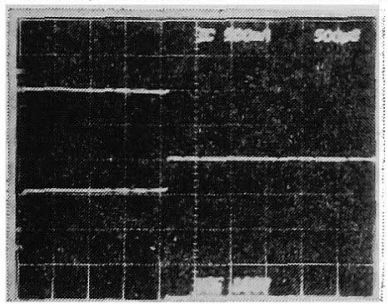

(a) Collector current (upper trace $I_{c}, 500 \mathrm{~mA} / \mathrm{div}$; time scale, $500 \mu \mathrm{s} / \mathrm{div}$ ) and collector-emitter voltage (lower trace $V_{C E}, 100 \mathrm{~V} / \mathrm{div}$; time scale, $500 \mu \mathrm{s} / \mathrm{div}$ ).

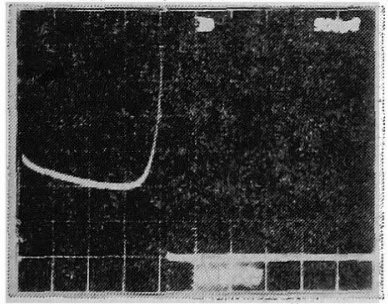

(b) Base current $I_{B}(2 \mathrm{~mA} /$ div; time scale, $500 \mu \mathrm{s} /$ div).

Fig. 3 Waveforms observed with the measuring circuit shown in Fig. 2.

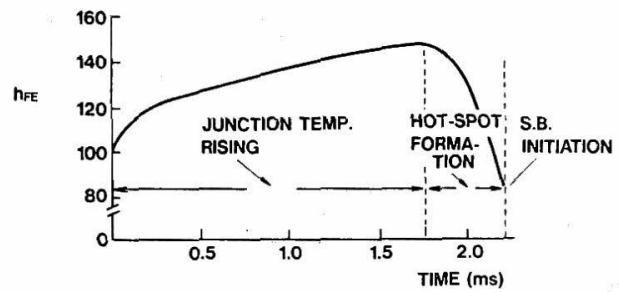

Fig. 4. Typical variations in current gain, $h_{F E}$, during high power dissipation.

interval and then shows a sudden increase after that. The end points of these waveforms correspond to the initiation of second-breakdown, which can be detected by a sudden fall in $V_{C E}$.

These characteristics of the base current can be related directly to variations in the current gain, because the collector current is held constant by the measuring circuit. Fig. 4 shows the variation in current gain obtained in a transformation from the waveform of the base current. The descending base current curve indicates that the increase in current gain is due to the rise in junction temperature before current concentration occurs. The time from the starting point to the minimum point of the base current, that is, the maximum point of current gain, is, therefore, thought to be the time during which the junction temperature is rising. The period after the minimum point of the base current is reached, during which the base current increases rapidly, is thought to be the period of thermal instability during which hot-spots are formed. The maximum point of current gain is, therefore, considered to be a point of change for junction temperature and current density, because thermal instability occurs at that point.

The beginnings of thermal instability, can, therefore, be discovered by detecting the minimum point of the base current, and the discovery of that point can be used to predict the occurence of second-breakdown. If the time required to reach the point is assumed to equal the time required for thermal instability to occur, that is the "delay time", then resistibility to "thermal-mode" second-breakdown can be determined from this delay time.

\section{Applications}

Resistibility to second-breakdown in power transistors with an $n^{+}-p-n-n^{+}$structure has been determined with the method described above. Some experimental results will be shown in the following figures.

\subsection{High temperature operation}

In power transistors for automotive use, ambient temperature is one of the most important conditions to be considered.

Fig. 5 shows the characteristics of a safe operating 


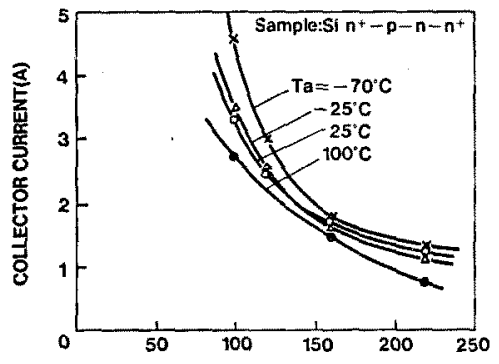

COLLECTOR-EMITTER VOLTAGE(V)

Fig. 5. The characteristics of a safe operating limit under various ambient temperature condition in a $n^{+} p-n-n^{+}$type power tran. sistor.

limit under various ambient temperature conditions in an $n^{+}-p-n-n^{+}$type power transistor for automotive use. This indicates that the limits of safe operation decrease as the ambient temperature increases.

\subsection{Hybrid integrated circuits}

The ambient temperature varies with changes in the thermal conditions of the device and substrate. Measurements of thermal-mode second-breakdown had, therefore, to be carried out also on samples in. stalled on a hybrid IC substrate.

Fig. 6 shows the resistibility characteristics of a power transistor chip mounted on a heat sink on an alumina substrate. In this case, the chip, heat sink and substrate were bounded together with a silicone adhesive agent. The heat sink was made of a copper plate as indicated in the figure. It was found that resis. tibility could be improved by increasing the size of the heat sink. However, it is neither necessary nor useful to increase the size of a heat sink indefinitely, because the area of the hot-spots that precede a second-breakdown is so small that heat flux cannot spread widely in lateral directions.

Fig. 6 also shows that the minimum points of resistibility are in a relatively low collector current region.

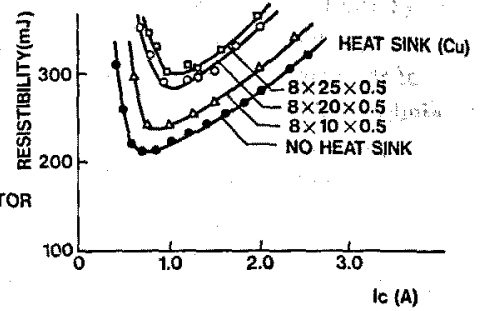

Resistibility characteristics of a power transistor chip mounted on a hybrid integrated circuit with heat sink.

These points must be considered when designing power transistors.

\subsection{Ignition systems}

The most severe automotive application of power transistors is, perhaps, in the ignition system, shown in Fig. 7 where a transistor must turn off an ignition coil with an inductive load greater than $5 \mathrm{mH}$. The current through the load is more than $5 \mathrm{~A}$. The amount of energy relased from the load is, therefore, extremely large, which tends to encourage degradation or failure in a power transistor. High voltage ignition surge is also applied to the power transistors.

Considering these severe requirements, we developed a power transistor with an $n^{+}-p-n-n^{+}$structure for automotive ignition systems. The $n^{+}-p-n-n^{+}$structure is constructed verticaly on a silicon chip $440 \mu \mathrm{m}$ thick. An $\mathrm{n}^{+}$-emitter diffused layer is constructed in the $\mathrm{p}$-base layer, which is fabricated in the $n$-original substrate. The thickness of the layers are $30 \mu \mathrm{m}$ and $50 \mu \mathrm{m}$, respectively. An $\mathrm{n}^{+}$-collector region was also thermally diffused onto the $n$-original substrate. The thickness of the $n$-and $n^{+}$-collector layers are, therefore, controlled by the diffusion depth of the $\mathrm{n}^{+}$-region. These thickness were optimized, taking into consideration the relation between resistibility to secondbreakdown and current gain, which will be described in a few moments. The emitter layer, and base-and emitter-electrodes were designed with the shapes

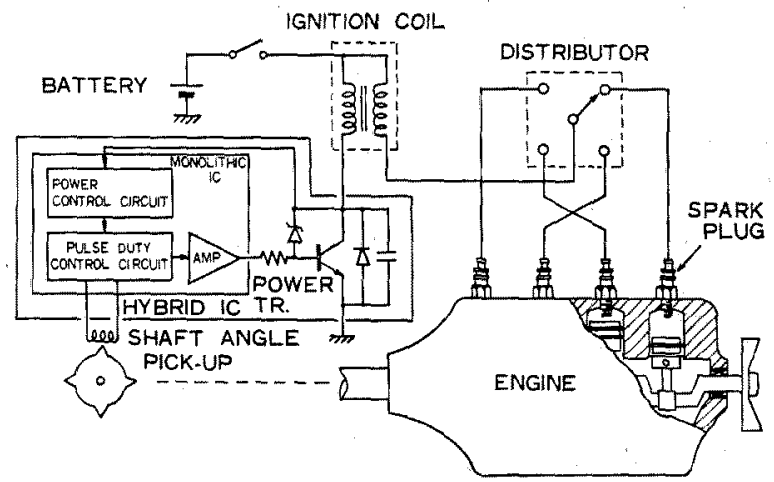

Fig. 7. Solid-state ignition system for automobiles. 


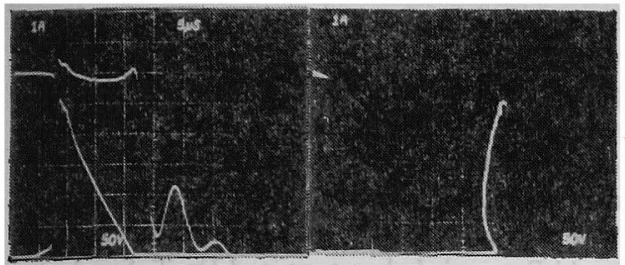

(a) Waveforms of $V_{C E}$ and $I_{C}$. (b) Locus of the operating point.

Fig. 8. Typical waveforms observed during turnoff operation in an ignition system.

shown in this figure in order to obtain uniform current distribution.

Fig. 8 shows typical waveforms observed during turn-off operations in an ignition system. In this case, the load inductance is $6 \mathrm{mH}$, and the current to turn-off is $6 \mathrm{~A}$. The current begins to fall a few micro-seconds after the base current is cut off. At the same time that the current begins to fall, the collector-to-emitter voltage begins to increase rapidly, but reaches an upper limit in the voltage sustaining region. The collector current decreases gradually, following the outline of the sustaining region determined by the characteristics of the transistor. Therefore, high power dissipation accompanies every turning off of the load, which makes the degradation of a transistor likely.

\subsection{Transisters with a high degree of resistibility to second-breakdown}

In order to investigate the characteristics of the degradation of resistibility that leads to second-breakdown, two structurally different types of $n^{+}-p-n-n^{+}$ transistors for automotive ignition systems were examined. The n-region of smale $A$ was $90 \mu \mathrm{m}$ long and that of sample $B$ was $50 \mu \mathrm{m}$.

Fig. 9 shows changes in resistibility leading to second-breakdown that were measured with the evaluation method mentioned above during repetitive switching of the inductive load. The energy applied in these experiments was about ten times as large as that applied in an acutual ignition system.

Resistibility was examined periodically during the test. A very large degradation was observed in sample $B$, but none in sample $A$, which had a longer n-region.

In order to produce a product that would have a

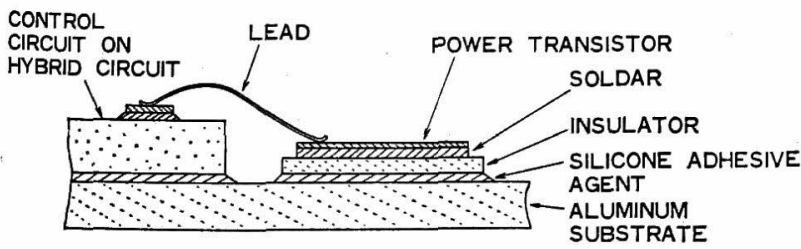

Fig. 10. Structure of solid-state ignition system integrated on hybrid IC.

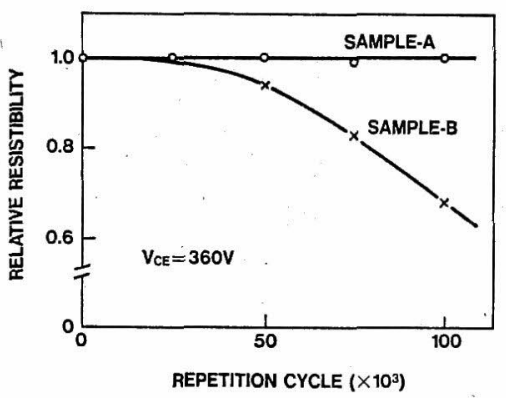

Fig. 9 Degradation characteristics of resistibility for two types of $n^{+}-p-n-n^{+}$transistors having different length $n$-regions.

good cost-performance ratio and was easy to handle, it was necessary to integrate the circuit for the solidstate ignition system on a hybrid IC and mount it in the ignition distributor.

The final structure of the solid-state ignition circuit is shown in Fig. 10. All circuit components, including a power transistor, were fabricated on an alumima base. A power transistor chip was mounted on an insulator plate with solder, and the insulator plate and alumina substrate were bound together with a silicone adhesive agent.

In this structure, thermal conductance between power transistor chip and alumina substrate must be considered.

Being indicated a moment ago, the length of the nregion in a $n^{+}-p-n-n^{+}$transistor has a large effect on the transistor's degree of resistibility to secondbreakdown. However, when resistibility is improved by lengthening the $\mathrm{n}$-region, unacceptable current gain losses occur.

Fig. 11 shows the relationship between current gain and resistibility, which were obtained from transistors with different length n-regions. In most automotive applications, the current gain losses that accompany

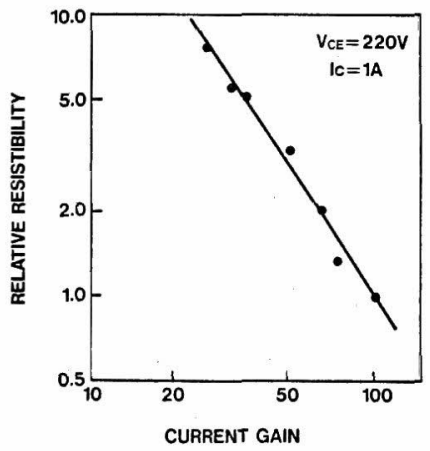

Fig. 11. Relationship between curren gain and resistibility in a type of $n^{+}-p-n-n^{+}$power transistors. 


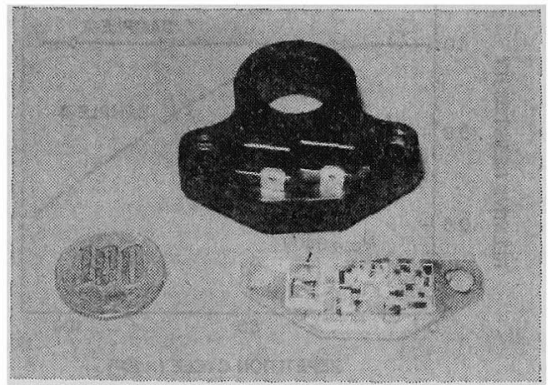

Fig. 12. Photograph of a solid-state ignition circuit designed to be built in an ignition distributor.

high resistibility to second-breakdown can be compensated for by adopting a "Darlington" transistor structure.

Fig. 12 is a photograph of a solid-state ignition circuit designed to be built in an ignition distributor. A power transistor and a control circuit are arranged on an alumina substrate. A pick-up coil, which is used to measure the angle through which an engine shaft rotates, is also built into the ignition module, and sends signals to the control circuit.

\section{Conclusions}

Many kinds of automotive equipment that includes power transistors with a high degree of second-breakdown resistibility in hybrid integrated circuits has been developed. Transistor and circuit design has been optimized with a new nondestructive method of evaluating resistibility to thermal-mode secondbreakdown which uses the characteristic change in the current gain of the transistor. The current gain variation involves the heating process of the collectorto-base junction and current concentrations at hotspots following thermal instability. The information on thermal-mode second-breakdown that has been presented here should allow the performance of bipolar transistors to be improved considerably. The new method of evaluation that has been developed is useful not only for device evaluation but also for circuit composition determination.

The author would like to thank Dr. E. Kaneko of the Hitachi Research Laboratory, and Dr. T. Suzuki and Dr. K. Hane of Keio Gijuku University, for their encouragement of this work.

\section{References}

(1) S. Y. Yu \& G. Thomas: "Trigger temperature of the second breakdown phenomenon", J. Appl. Phys., 48, 1166 (1977)

(2) C. G. Thornton \& C. D. Simons: "A new high current mode of transistor operation", IRE Trans. Electron Devices, ED-5, 6 (1958)

(3) K. Koyanagi, K. Hane \& T. Suzuki: "Boundary conditions between current mode and thermal mode second breakdown in epitaxial planar transistors", IEEE Trans. Electron Devices, ED-24, 672 (1977)

(4) W. P. Bennett \& R. A. Kumbatovic: "Power and energy limitations of bipolar transistors imposed by thermal-mode and current mode second-breakdown mechanisms", ibid, ED-28, 1154 (1981)

(5) P. L. Hower \& V.G. Reddi: "Avalanche injection and second breakdown in transistors", ibid., ED-17, 320 (1970)

(6) R. M. Scarlett \& W. Shockley: "Secondary breakdown and hot-spots in power transistors", IEEE Int. Con. Rec., 11, (1963)

(7) P. L. Hower, D. L. Blackburn, F. F. Oettinger \& S. Rubin: "Stable hot-spots and second breakdown in power transistors", 1976 IEEE Power Electronics Specialists Conf., p. 234 (1976)

(8) T. Sasayama \& K. Ohnishi: "Second breakdown in germanium power transistors", Hitachi Hyoron, 49, 1184 (1967)

(9) G. M. Ford: "Collector to emitter breakdown reiated to thermal runaway in homogeneous base germanium power transistors", Solid-State Des., 4, 29 (1963)

(10) T. Sasayama: "Properties and their applications of current gain variation preceding to the second breakdown in power transistors", Trans. IECE of Japan, E-62, 858 (1979)

(11) T. Sasayama: "A method to evaluate the second breakdown resistance of power transistors", IEEE Trans. Inst. and Meas., IM-29, 48 (1980) 